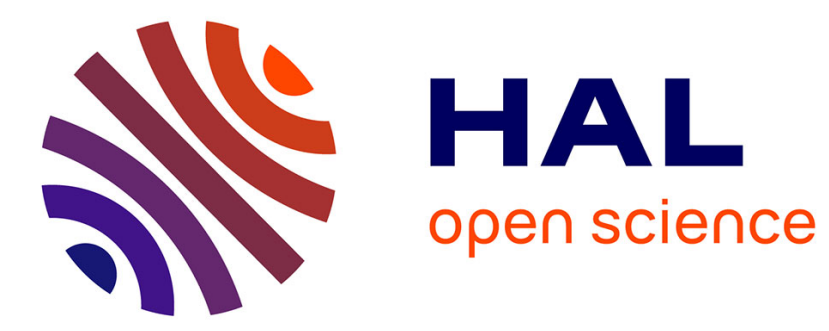

\title{
La surcomposition verbale et ses emplois en français
}

Denis Apothéloz

\section{To cite this version:}

Denis Apothéloz. La surcomposition verbale et ses emplois en français. A. Patard, R. Peltola, E. Roussel. Cross-Linguistic Perspectives on the Semantics of Grammatical Aspect, 30, BRILL, pp.1337, 2019, Cahiers Chronos, 978-90-04-39933-4. 10.1163/9789004401006_003 . hal-02077549v2

\section{HAL Id: hal-02077549 \\ https://hal.science/hal-02077549v2}

Submitted on 27 Apr 2019

HAL is a multi-disciplinary open access archive for the deposit and dissemination of scientific research documents, whether they are published or not. The documents may come from teaching and research institutions in France or abroad, or from public or private research centers.
L'archive ouverte pluridisciplinaire HAL, est destinée au dépôt et à la diffusion de documents scientifiques de niveau recherche, publiés ou non, émanant des établissements d'enseignement et de recherche français ou étrangers, des laboratoires publics ou privés. 
A paraître dans :

Adeline Patard, Rea Peltola \& Emmanuelle Roussel (eds) :

Cross-Linguistic Perspectives on the Semantics of Grammatical Aspect.

Leiden / Boston : BRILL-RODOPI, 2019, 13-37.

(Cahiers Chronos 30)

\title{
La surcomposition verbale et ses emplois en français ${ }^{1}$
}

\author{
Denis Apothéloz \\ Université de Lorraine, ATILF
}

\begin{abstract}
Résumé
Cet article part du constat selon lequel le passé surcomposé français, dans l'immense majorité de ses emplois, peut recevoir deux types d'interprétations : un type qualifié ici de RÉSULTATIF, permettant d'exprimer un état résultant localisé dans le passé ; et un type qualifié d'EXISTENTIEL, exprimant la valeur de parfait d'expérience (au sens de Zandvoort 1932). L'auteur montre, en analysant de nombreux exemples, que ces deux types d'interprétations se retrouvent dans tous les temps surcomposés du français, y compris les participes, et que cette opposition est donc un élément clé du système des temps composés (parfaits) de cette langue. L'article présente également diverses observations et hypothèses concernant les fonctions et les contextes d'utilisation des formes surcomposées.
\end{abstract}

Mots-clés

temps surcomposés, parfait, parfait d'expérience, état résultant

\begin{abstract}
The starting point of this article is the observation that in the vast majority of its uses, the French double perfect (passé surcomposé) can receive two kinds of interpretations: one we shall call RESULTATIVE, which expresses a resulting state located in the past; the other, called EXISTENTIAL, expressing the perfect of experience (in the sense of Zandvoort 1932). The author shows with numerous examples that all the French double perfect forms - including the participles - can achieve these two types of interpretations, and that this opposition is therefore a key feature of the French system of compounded tenses (perfects). Furthermore, the article discusses some evidence and assumptions about the functions and contexts of use of double perfect tenses.
\end{abstract}

Keywords

double perfect tenses, perfect, perfect of experience, resulting state

\section{Introduction}

Dans le champ des études sémantiques portant sur l'aspect grammatical, il existe à ce jour une somme considérable de travaux consacrés à la sémantique des parfaits. Concernant la langue française, plusieurs études ont été consacrées ces dernières années au passé composé, souvent dans une perspective diachronique et dans le cadre d'une confrontation avec le passé simple (e.g. Caron \& Liu 1999 ; Caudal \& Vetters 2007 ; Vetters 2010 ; Schøsler 2012 ; Apothéloz 2017). Dans ce panorama, il apparaît que les formes surcomposées, certes moins fréquentes et souvent ignorées par les grammaires d'usage, ont longtemps fait figure de parent

\footnotetext{
${ }^{1}$ Un chaleureux merci à Marine Borel (Université de Fribourg \& Université de Lorraine), qui a relu minutieusement une version antérieure de cet article et m'a fait bénéficier de son expertise dans le domaine de la surcomposition verbale. Je remercie également les deux relecteurs anonymes pour la pertinence de leurs remarques, notamment concernant l'interprétation de certains exemples.
} 
pauvre, en dépit de quelques publications importantes (comme Foulet 1925 ; Cornu 1953 ; Stefanini 1954 ; Carruthers 1994).

Depuis quelques années, on observe cependant un regain d'intérêt pour le phénomène de la surcomposition verbale en français (e.g. Carruthers 1998, 1999 ; Paesani 2001 ; Sthioul 2006 ; Wilmet 2009; Apothéloz 2010, 2012 ; Melchior 2012; de Saussure \& Sthioul 2012; Havu 2013 ; Borel 2017). Cette tendance paraît d'ailleurs dépasser le domaine des études romanes (e.g. Schaden 2009 ; Haß 2016). Le présent article s'inscrit dans ce courant. Son objectif est relativement simple: on partira de l'observation suivant laquelle le passé surcomposé français (qui est, de l'avis général, la forme surcomposée la plus fréquente dans cette langue) est apte à produire deux valeurs clairement distinctes, valeurs qui seront décrites en détail. On formulera ensuite l'hypothèse que ces deux valeurs expliquent l'immense majorité des emplois des autres temps surcomposés, et on mettra cette hypothèse à l'épreuve des faits. Cette étude peut donc être regardée comme une contribution à la sémantique des parfaits du français.

\section{Les deux emplois du passé surcomposé}

En français contemporain, les données indiquent très clairement que les emplois du passé surcomposé peuvent être classés dans l'un des deux types suivants ${ }^{2}$ :

(i) Un TYPE RÉSULTATIF, dont la valeur ainsi que les contextes d'occurrence sont relativement aisés à identifier. De façon caractéristique, les énoncés où on rencontre ce passé surcomposé désignent un état résultant localisé dans le passé. On trouve le type résultatif dans les contextes suivants :

- Dans des subordonnées temporelles introduites par des conjonctions ou expressions conjonctives comme: quand, lorsque, aussitôt que, sitôt que, après que, dès que, une fois que, jusqu'à ce que, depuis que, le/du jour où, au moment où, etc. :

(1) Je l'ai aperçue depuis l'autre côté de la rue et, dès que je l'ai eu aperçue, j'ai eu envie d'elle à en crier. (L. Malet, 1969)

(2) [...] quoiqu'il ait eu des qualités vraiment grandes et extraordinaires, il a travaillé à les cacher et à les étouffer autant qu'il a pu depuis qu'il a eu quitté la cour, par la manière simple et commune dont il a toujours vécu depuis. (J.-F. Michaud, J.-J.-F. Poujoulat, 1837)

- Dans des subordonnées dites inverses en : ne...pas... que, ne... pas plutôt (écrit parfois plus tôt)... que, ne... pas encore... que, à peine... que, etc.

(3) [...] pour ce banquet, je ne l'ai pas eu plutôt engagé, qu'il s'est rendu tout de suite à mon invitation. (Platon, Lettres, Trad. Victor Cousin)

(4) [...] à peine a-t-il eu fait quelques pas que nous l'avons vu tomber auprès de la porte du salon. (G. Sénac de Meilhan, 1797)

Dans ces deux premiers types de contextes, l'état résultant désigné par la forme surcomposée localise temporellement une autre situation, elle-même exprimée par une forme verbale le plus souvent au passé composé, comme c'est le cas dans les exemples (1)-(4).

- Le type résultatif se rencontre également sous l'incidence d'un adverbial quantifiant l'intervalle temporel entre une situation précédemment évoquée et la situation désignée par la forme surcomposée. Cet adverbial (bientôt, en cinq minutes, rapidement, vite, etc.) indique la plupart du temps que cet intervalle a été bref ou plus court que prévu (cf. ex. 5 et 6). Dans sa

\footnotetext{
${ }^{2}$ Pour des observations analogues dans le domaine roman, voir par exemple Squartini (1998).
} 
forme minimale, l'adverbial peut prendre la forme d'une simple expression de consécution temporelle, comme et puis dans l'exemple (7):

(5) Il filait, filait sans bouger. En cinq minutes, il a eu disparu. (Corresp. Alain-Fournier - J. Rivière, 1910)

(6) Et pourtant, on a eu fait très rapidement, là encore, le tour de la question. (B. Bayon, 1987)

(7) Et puis, il a eu fini de bâfrer. Pas trop tôt. Il était minimum sept heures. (B. Bayon, 1987)

- Exceptionnellement cet élément de liaison peut être absent, comme dans (8). Mais la désignation de l'état résultant par la forme surcomposée n'en marque pas moins une progression temporelle :

(8) Je m'y suis remise avec ardeur, il fallait avoir fini le 15. J'ai eu fini, et sans trop manquer ma sauce, à ce que j'espère. (G. Sand, 1846)

Les cas illustrés par (7) et (8), dans lesquels le surcomposé est introduit par un simple marqueur de consécution, ou n'est pas introduit du tout, sont rares et ne se rencontrent pratiquement qu'avec le verbe finir (Borel, comm. pers.). Il semble y avoir une affinité particulière entre ce verbe et le passé surcomposé en emploi résultatif, comme le notait Olsson (1971) dans sa thèse sur les subordonnées temporelles.

Cet emploi du passé surcomposé est généralement considéré par les grammairiens comme standard, par quoi il faut comprendre qu'il n'est pas limité à une aire géographique particulière. Du point de vue de sa signification aspectuelle, c'est un passé accompli au sens traditionnel du terme (e.g. Imbs 1960), à l'image du passé antérieur. Son emploi intervient systématiquement dans des séquences narratives.

(ii) Un TYPE EXISTENTIEL, dont la signification est celle d'un parfait d'expérience (Apothéloz 2010). La signification de parfait d'expérience a été mise en évidence par Zandvoort (1932) à propos du Present perfect et du Past perfect de l'anglais. Elle est aussi désignée par 1'expression de parfait existentiel (McCawley 1971, 1981), ou encore de passé indéfini (Leech 1971), et est régulièrement évoquée pour rendre compte de l'un des emplois du Present perfect (Comrie 1976; Fenn 1987; Michaelis 1998; Mittwoch 2008). On peut décrire la valeur sémantique du passé surcomposé de type existentiel comme suit : l'énoncé où figure cette forme indique que la situation désignée est advenue une fois au moins dans un intervalle temporel localisé dans le passé. En voici deux exemples :

(9) Oui, j'ai eu écrit des lettres d'amour à mon chéri et il aimait bien les recevoir. Maintenant je lui écrit [sic] des petits mots doux ou je lui envoie des mails lorsqu'il est au boulot. (Forum internet, $2005)^{3}$

(10) Mais avez-vous eu été confrontés aux flics une fois dans votre vie ? Moi oui et pourtant je ne suis ni voleur ni délinquant. (Site du journal Libération, 2009, message d'un lecteur)

J'ai eu écrit des lettres d'amour à mon chéri, et avez-vous eu été confrontés aux flics peuvent être paraphrasés respectivement de la façon suivante :

- 'il m'est arrivé (une ou plusieurs fois) d'écrire des lettres d'amour à mon chéri',

- 'vous est-il arrivé (une ou plusieurs fois) d'être confrontés aux flics ?'.

Dans l'exemple (10), l'expression dans votre vie est une spécification de l'intervalle temporel mentionné ci-dessus.

Le type existentiel se distingue du type résultatif par le fait qu'il n'est pas associé à des environnements syntaxiques particuliers. Il est vrai qu'il apparaît le plus souvent dans des propositions principales ou indépendantes. Mais, contrairement à ce qui a parfois été écrit, il

\footnotetext{
${ }^{3}$ Les exemples sont reproduits avec leur orthographe d'origine.
} 
n'est pas incompatible avec les subordonnées, y compris les subordonnées temporelles. C'est ce que montrent les exemples ci-dessous :

(11) Effectivement, ça m'a échappé, parce que je ne renomme jamais les fichiers dans un albums ou si je l'ai eu fait, toute [sic] les photos provenaient probablement d'un même dossier. (Blog, 2012)

(12) quand on a eu fait des sorties en catamaran on a vu beaucoup de marsouins (Oral, Suisse romande, 2015, in Borel, à par.)

Si je l'ai eu fait signifie ici 's'il m'est arrivé de le faire', et quand on a eu fait des sorties en catamaran, 'quand il est arrivé qu'on fasse des sorties en catamaran'.

On ne rencontre en principe le type existentiel que dans les territoires à substrat occitan et franco-provençal, c'est-à-dire dans la partie méridionale de la France ainsi que dans la Suisse francophone (Suisse Romande). Pour cette raison, il est parfois qualifié de régional, le type résultatif étant alors qualifié de général (Jolivet 1986). Il est par ailleurs régulièrement stigmatisé par les instances normatives. À la différence du type résultatif, le type existentiel appartient clairement au registre argumentatif.

Morphologiquement, les formes du passé surcomposé sont construites comme suit : les verbes à auxiliaire avoir ont une forme du type a eu fait, qui admet les deux interprétations (résultative et existentielle). Les verbes pronominaux ont une forme du type s'est eu levé, forme qui admet également les deux interprétations. Quant aux verbes à auxiliaire être nonpronominaux, ils ont la propriété remarquable de présenter deux formes : l'une de type a été arrivé, toujours résultative; l'autre de type est eu arrivé, toujours existentielle. Cette particularité avait déjà été notée par Foulet (1925), qui proposait d'appeler parfaits surcomposés les formes surcomposées existentielles. Cette spécialisation, ainsi que d'autres propriétés formelles qu'il n'est pas possible de développer ici, pourraient être un argument pour considérer que les types résultatif et existentiel constituent deux temps verbaux distincts (sur cette hypothèse, voir Borel à par. $)^{4}$.

Du point de vue diachronique, l'histoire des formes surcomposées et de leurs emplois est assez confuse. Ces formes semblent attestées dès le XII ${ }^{\mathrm{e}}$ s. (Wagner 1954). Mais la distinction des deux types décrits ci-dessus est probablement plus tardive. Les recherches menées par Borel (2017) aboutissent à la conclusion que cette dissociation s'est vraisemblablement produite entre le $\mathrm{XV}^{\mathrm{e}}$ et le $\mathrm{XVI}^{\mathrm{e}} \mathrm{s}$.

En français standard, où seul existe le type résultatif, la signification de parfait d'expérience des exemples (9) à (12) serait exprimée au moyen d'un passé composé, en éventuelle association avec l'adverbe déjà, avec un adverbe quantifieur de fréquence (rarement, parfois, souvent, jamais) ou quantifieur d'occurrence (plus d'une fois, plusieurs fois, etc.) $)^{5}$. Elle peut également être exprimée par le passé composé dans les territoires où on rencontre le type existentiel du passé surcomposé. La différence entre les deux temps est alors que le passé composé ne grammaticalise pas cette signification. Elle ne lui advient qu'en présence de certains indices contextuels (par exemple, la présence d'un adverbe quantifieur de fréquence ou d'occurrence). En revanche, pour les sujets parlants dont la grammaire inclut le passé surcomposé existentiel, celui-ci fonctionne comme une forme spécifiquement dédiée à l'expression du parfait d'expérience. Ce temps verbal a donc, pour ces sujets parlants, un statut d'overt category au sens de Whorf (1956). Il permet d'assurer une telle interprétation, dans des contextes où un passé composé pourrait être ambigu. En un mot, il la grammaticalise. Pour le montrer, examinons l'extrait suivant :

(13) on adore rire ensemble/ on fait des soirées ensemble/ on est eu parti en vacances ensemble/ (Oral, locutrice neuchâteloise, 2009. '/' indique une intonation montante)

\footnotetext{
${ }^{4}$ Sur cette question, par ailleurs controversée, voir également Jolivet (1986) et de Saussure \& Sthioul (2012).

${ }^{5}$ Ces adverbes permettent alors d'assurer l'interprétation de parfait d'expérience, interprétation qui serait, sinon, en concurrence avec une interprétation de prétérit.
} 
Pour un locuteur dont la grammaire inclut le passé surcomposé existentiel, cet énoncé signifie 'il nous est arrivé (une fois au moins) de partir en vacances ensemble', et ne peut signifier que cela. Or, pour tout locuteur francophone, un passé composé pourrait ici être ambigu :

(14) on est parti en vacances ensemble

Certes, le contexte de (13) favorise vraisemblablement une interprétation existentielle du passé composé de (14); mais il n'en demeure pas moins que (14), dans le contexte de (13), est à même de désigner :

- soit une occurrence singulière, définie et localisée dans le temps, de la situation consistant à « être parti en vacances ensemble », comme ce serait le cas dans (14') :

(14') l'été dernier on est parti en vacances ensemble

- soit un nombre indéterminé d'occurrences de ce type de situation, tout en signifiant qu'elle est advenue une fois au moins (interprétation de parfait d'expérience, donc existentielle).

On notera que cette lecture existentielle serait la seule possible si le passé composé était sous l'incidence de l'adverbe déjà ou d'un adverbial quantifieur de fréquence ou d'occurrence :

(15) on est \{déjà / souvent / parfois / rarement / plusieurs fois /...\} parti en vacances ensemble

Il est intéressant de noter que dans ces formulations, l'adverbial porte sur le composant existentiel de l'énoncé, comme le montrent les paraphrases: 'il est $\{$ déjà / souvent / parfois /... $\}$ arrivé qu'on parte en vacances ensemble ${ }^{\prime 6}$.

\section{Principales propriétés du passé surcomposé de type existentiel}

Le type existentiel se caractérise par les propriétés principales suivantes :

(i) La proposition qui comporte ce type de surcomposé a une signification qui est dominée par une ASSERTION D'ADVENUE (ou d'existence). Cette signification correspond, dans les paraphrases données ci-dessus, à l'expression il est arrivé (une ou plusieurs fois) que... ${ }^{7}$

(ii) Cette assertion d'advenue est associée à un INTERVALLE DE VALIDATION, expression que j'emprunte à Desclés (1997). Pour qu'elle puisse être validée, en effet, il faut que cette assertion soit associée à un domaine, autrement dit à un intervalle temporel. Cet intervalle peut être implicite (cas le plus fréquent) ou explicite. Dans l'exemple (9) - répété ci-dessous il demeure implicite; la seule information qu'on ait à son sujet est qu'il est antérieur au moment de l'énonciation. Dans l'exemple (10), en revanche, il est indiqué au moyen de l'expression dans votre vie, comme nous l'avons vu plus haut.

\footnotetext{
${ }^{6}$ Concernant le Present perfect, Leech $(1971:$ \$56) indiquait que, quand la signification de parfait d'expérience n'est pas accompagnée d'un adverbial, l'auxiliaire tend, en langue parlée, à être produit avec une certaine emphase prosodique. Intuitivement, il me paraît que cette observation vaut également pour le français ; mais il n'existe, à ma connaissance, aucune étude empirique sur la prosodie des parfaits d'expérience du français. A noter quand même que Foulet (1925 : 232) observait, à propos d'un exemple de surcomposé existentiel, que le $e u$ était prononcé « avec un fort accent »!

${ }^{7}$ Croft (2012 : 163) propose une glose un peu différente, mais en tous points équivalente : 'there exists at least one past situation of...' [suit la désignation de la situation concernée]. Foulet (1925) déjà avait noté que le tour impersonnel il est arrivé que fournit une bonne paraphrase de certains emplois du passé surcomposé.
} 
(9) Oui, j'ai eu écrit des lettres d'amour à mon chéri et il aimait bien les recevoir. Maintenant je lui écrit [sic] des petits mots doux ou je lui envoie des mails lorsqu'il est au boulot. (Forum internet, 2005)

(10) Mais avez-vous eu été confrontés aux flics une fois dans votre vie ? Moi oui et pourtant je ne suis ni voleur ni délinquant. (Site du journal Libération, 2009, message d'un lecteur)

Quand le type existentiel est exprimé au moyen d'un passé composé, la borne terminale de l'intervalle de validation coïncide par défaut ${ }^{8}$ avec le moment de l'énonciation (désigné ciaprès par $\mathrm{T}_{0}$ ). A cet égard, le passé composé français fonctionne comme le Present perfect anglais (cf. Comrie 1976). Théoriquement, cette borne terminale devrait donc, dans le cas du passé surcomposé, être localisée avant $\mathrm{T}_{0}$, donc dans le passé. Or, les données montrent que ce n'est pas toujours le cas (Apothéloz 2012). On observe en effet une certaine variation dans la façon dont cet intervalle est traité. Cette variation concerne la borne terminale de l'intervalle. Ainsi, dans (9), où la locutrice met explicitement en contraste ce qu'elle faisait avant et ce qu'elle fait maintenant, la borne terminale de l'intervalle de validation paraît localisée dans le passé. Il en résulte que, dans ce contexte, on ne peut pas inférer de l'énoncé J'ai eu écrit des lettres d'amour... que les situations qu'il désigne sont susceptibles de se reproduire à $\mathrm{T}_{0}$. Il en va différemment dans (10), où rien ne paraît empêcher que la borne terminale de l'intervalle de validation coïncide avec $\mathrm{T}_{0}$. Pour cette raison, la question Avezvous eu été confrontés aux flics une fois dans votre vie n'exclut pas qu'on en infère la possibilité d'autres confrontations de ce type. (Pour une discussion de ce problème, voir de Saussure \& Sthioul 2012 ainsi que Borel à par.)

On notera que, quand la borne terminale de son intervalle de validation coïncide avec $\mathrm{T}_{0}$, le passé surcomposé de type existentiel ne se distingue pas du passé composé du même type.

(iii) La troisième propriété du passé surcomposé existentiel, et des parfaits d'expérience en général, est que la localisation temporelle de la (ou des) situation(s) dont l'énoncé dit qu'elles ont existé est indéfinie, hormis bien entendu le fait qu'elles sont situées à l'intérieur de l'intervalle de validation. Cela explique l'appellation que Leech (1971) a donné au Present perfect existentiel (indefinite past).

(iv) Enfin, le passé surcomposé existentiel est associé à un type particulier de résultativité, donc d'état résultant, qui est propre aux parfaits d'expérience. Il s'agit d'une résultativité indirecte, très différente de la traditionnelle valeur d'accompli, et que Desclés (2017) appelle ÉTAT D’EXPÉRIENCE. Dans le cas du passé surcomposé, cet état d'expérience est validé à (et depuis) $\mathrm{T}_{0}$. Dans les formes de type avait eu fait, son moment de validation est localisé dans le passé. Pour rendre compte de la complexité du fonctionnement référentiel des parfaits d'expérience, Gosselin (2017) considère qu'ils réfèrent aussi bien au(x) moment(s) où la situation est advenue qu'à l'état d'expérience.

\section{Qu'en est-il des autres temps surcomposés?}

Le passé surcomposé n'est bien sûr pas la seule forme surcomposée qu'on rencontre en français. La question se pose donc de savoir si les deux types qui viennent d'être décrits sont valables également pour ces autres temps surcomposés.

\subsection{Quelques données quantitatives}

\footnotetext{
${ }^{8}$ C'est-à-dire à défaut d'informations spécifiant l'une et/ou l'autre de ses bornes, comme le ferait par exemple l'expression depuis notre dernière rencontre.
} 
De façon générale, l'existence d'autres temps surcomposés est reconnue depuis longtemps. Ainsi, l'une des premières grammaires du français, le Traité de Louis Meigret (1550), fait état de formes comme ai eu fait, ai été arrivé, mais aussi avais eu fait (p. 124), aurai eu fait (p. 133) ou encore aurais eu fait et eusse eu aimé (p. 95). A l'époque classique, les temps surcomposés, du moins certains, sont souvent mentionnés par les grammairiens, même si ces derniers paraissent parfois davantage motivés par le souci de présenter un système complet et méthodique (comme Beauzée 1765), que par le souci de rendre compte de formes réellement attestées.

Cependant, il est extrêmement difficile d'établir des informations fiables concernant la fréquence des emplois des temps surcomposés. Les variantes existentielles, en particulier, probablement parce qu'elles sont souvent stigmatisées par le discours normatif, peuvent être d'un usage tout à fait courant chez certains locuteurs, et totalement absentes quand ces mêmes locuteurs s'expriment par écrit, en particulier dans des documents destinés à être imprimés. A cet égard, il ne fait pas de doute que la prolifération des écrits électroniques à laquelle on assiste depuis une vingtaine d'années a considérablement changé les données du problème, et permet d'accéder à des faits qui, auparavant, auraient passé complètement inaperçus.

En dépit de ces difficultés, il peut être utile de présenter ici quelques données quantitatives. Celles que j'ai réunies dans le Tableau 1 m'ont été transmises par M. Borel et constituent l'essentiel du corpus de sa thèse (Borel à par.). Ce corpus comporte à l'heure actuelle 5296 exemples attestés, allant $d u \mathrm{XV}^{\mathrm{e}}$ au XXI ${ }^{\mathrm{e}}$ s. Pour éviter les problèmes de dénomination que posent ces temps verbaux, j'adopte ici la méthode utilisée par cette auteure, consistant à identifier les temps surcomposés au moyen d'un exemple canonique utilisant le verbe faire. Ils sont ordonnés de gauche à droite selon une fréquence décroissante.

\begin{tabular}{|c|c|c|c|c|c|c|c|c|}
\hline a eu fait & $\begin{array}{c}\text { avait eu } \\
\text { fait }\end{array}$ & $\begin{array}{l}\text { aurait } \\
\text { eu fait }\end{array}$ & $\begin{array}{c}\text { ayant eu } \\
\text { fait }\end{array}$ & $\begin{array}{c}\text { aura eu } \\
\text { fait }\end{array}$ & $\begin{array}{c}\text { avoir eu } \\
\text { fait }\end{array}$ & $\begin{array}{l}\text { ait eu } \\
\text { fait }\end{array}$ & $\begin{array}{l}\text { autres } \\
\text { formes }\end{array}$ & \\
\hline $77.4 \%$ & $6.1 \%$ & $4.4 \%$ & $3.3 \%$ & $2.9 \%$ & $2.7 \%$ & $1.8 \%$ & $1.4 \%$ & $100 \%$ \\
\hline
\end{tabular}

Il apparaît immédiatement que les formes en a eu fait (passé surcomposé) sont de très loin les plus fréquemment utilisées, puisqu'elles représentent plus des trois quarts des formes surcomposées répertoriées.

\subsection{Exemples}

L'examen des données indique que la réponse à la question posée plus haut est positive : les formes surcomposées du français sont toutes susceptibles de réaliser le type résultatif et le type existentiel, à l'exceptions de eut eu fait et de eût eu fait, formes rarissimes et toujours résultatives ${ }^{10}$. Je donne ci-dessous 2 exemples de chacun des deux types, pour les formes suivantes : avait eu fait, aurait eu fait, aura eu fait, ait eu fait, ayant eu fait, avoir eu fait. Les formes verbales surcomposées sont en gras. Les autres expressions en gras concernent des commentaires qui seront faits plus loin.

\section{Avait eu fait}

\section{Type résultatif}

\footnotetext{
${ }^{9}$ C'est-à-dire au moment où ces lignes sont rédigées (juillet 2018).

${ }^{10}$ On trouve dans Holtus (1995) plusieurs exemples de formes surcomposées appartenant au type existentiel, mais elles ne sont pas diagnostiquées comme telles par l'auteur.
} 
(16) [...] nos soupçons ont acquis un nouveau degré de certitude, lorsqu'il nous a été dit par les assistans, et confirmé par le malade lui-même, que ces accidens s'étaient manifestés peu de temps après qu'il avait eu mangé d'une soupe dont la saveur lui avait paru âcre et austère. (J. Briand, J.X. Brosson, 1828)

(17) Étant parfaitement renseigné sur l'état des lieux, il avait eu vite fait de voir comment il lui faudrait s'y prendre pour passer sans être aperçu par ceux du poste, ce qui n'était pas très difficile ; et, une nuit donc, il était parti [...]. (C.-F. Ramuz, 1925)

\section{Type existentiel}

(18) faut aussi se remettre dans le contexte que j'étais un un vieux de de de je sais plus [...] quarante-six ans qui qui avait eu fait du cheval mais qui n'avait plus du tout la musculature d'un cavalier donc hein $[\ldots]$ et pis plus les réflexes (OFROM, Corpus oral de français de Suisse romande, 2012)

(19) [Il est question d'un chien mort]

Je l'avais enveloppé dans un drap tout blanc, son linceul, et nous avions pris rendez-vous au centre d'incinération pour animaux, je voulais ses cendres pour les disperser dans la nature. Au milieu du lac, avais-je d'abord imaginé, mais Billy n'aimait pas se baigner. Il m'était eu arrivé de le doucher, très rarement, événement très pénible pour tous deux. (Blog, 2011)

\section{Aurait eu fait}

\section{Type résultatif}

(20) Et quand celui-ci aurait eu fini de répondre, il lui aurait annoncé qu'à compter de cet instant le contact était rompu entre eux. (H. Mankell, 2012)

(21) [...] elle serait tombée d'elle-même, dès qu'on lui aurait eu coupé ses communications avec Constantinople. (F. de Beaujour, 1829)

\section{Type existentiel}

(22) [Une femme décrit les effets des médicaments neuroleptiques]

Je me levais au milieu de la nuit car je voulais aller dehors me promener bref des choses que jamais j'aurais eu fait dans mon état normal. (Blog, 2014)

(23) Aussi, monsieur - reprit M. Pipelet - j'aurais eu commis des crimes affreux, que je n'aurais pas eu un sommeil plus bourrelé. (E. Süe, 1843)

\section{Aura eu fait}

\section{Type résultatif}

(24) Prenez une demi-feuille de papier uni, que vous oindrez d'huile douce. Après que cette onction aura eu fait son effet, pendant une ou deux minutes, laissez l'huile suinter à travers le papier ; (Manuel d'économie rurale et domestique, 1820, trad. de l'anglais)

(25) Nil se dit que c'était un geste typique d'adolescente amoureuse. " Quand elle aura vingt-cinq ans, et qu'un régiment de cavalerie lui aura eu défilé sur le corps, elle ne baisera plus la main de son amant du jour, ou si elle le fait, ce ne sera pas élan, mais calcul. » (G. Matzneff, 1981)

\section{Type existentiel}

(26) tu imagines pas le nombre de fois dans ma vie où j'aurai eu entendu des conversations téléphoniques qui parlaient de moi (Oral, Suisse romande. In : Borel, à par.)

(27) [Il est question de personnes qui se suicident]

Elles tentent d'abord de résister, comme elles l'auront eu fait souvent tout au long de leurs vies, 
elles ont justement l'expérience des difficultés et savent se battre, elles sont, à ce sujet, bien plus aguerries que la moyenne des gens. (Presse internet, 2013)

\section{Ait eu fait}

\section{Type résultatif}

(28) Alors pendant que j'étais dans le bois, la mère s'est mise à voyager à la rivière avec une chaudière dans chaque main [...], jusqu'à ce qu'elle ait eu fini de remplir un quart [...]. (L. Hémon, 1913)

(29) Le débat sur ce point a pris fin après que j'aie eu pris la parole, et que bon nombre d'honorables sénateurs avaient posé des questions [...]. (Parlement du Canada, 2000)

\section{Type existentiel}

(30) [Il est question d'une manière particulière de recharger les armes à feu avec de la poudre] Que des anciens l'aient eu fait, c'est une chose.... le recommander c'en est une autre !! Moi j'ai aussi connu des poudreux qui rechargeaient à la poire avec la clope au bec, est-ce pour autant qu'il faut le faire ??? (Forum internet, 2009)

(31) Le pire que j'aie eu vu c'est un livreur français avec un semi-remorque qui devait aller livrer qqch à Mex [Localité près de Lausanne]. (Forum internet, 2005)

\section{Ayant eu fait}

\section{Type résultatif}

(32) Ayant eu terminé de mettre des pneus assez tard ce soir, je n'ai eu l'occasion que de faire quelques centaines de mètres avec et c'est une bonne surprise. (Blog, 2011)

(33) [Il est question de la série des films Massacre à la tronçonneuse] Ce film avait été censuré sous le règne de Giscard D'Estaing, par un Comité qui avait empêché sa sortie en salle. Ayant eu vu le second, un chef d'œuvre d'humour noir, je me faisais une joie de regarder le premier de cette série, l'« original ». (Forum internet, 2004)

\section{Type existentiel}

(34) $[\ldots]$ j'ai une hantise des antidépresseurs ayant eu fait déjà plusieurs essais négatifs [...]. (Forum internet, 2006)

(35) [...] j'aurais aimé échanger avec des personnes ayant eu eu des traumatismes similaires [...]. (Forum internet, 2007)

\section{Avoir eu fait}

\section{Type résultatif}

(36) C'est pourquoi après avoir eu terminé la Wirtschaftsmittelschule à Berne, il a suivi une formation pour devenir webmaster. (Presse internet, 2013)

(37) J'ai fréquenté les forums maternité d'Auféminin avant-pendant-et après avoir eu eu mes filles (6 et 3.5 ans), mais là ça faisait un moment que je ne postais plus, en tout cas dans les forums bébés ! (Forum internet, 2009)

\section{Type existentiel}

(38) The Daily Mail vient cependant de lâcher une petite bombe : selon le journal, Nicole Kidman aurait enfin admis avoir eu fait des injections de Botox ces dernières années. (Presse internet, 2011) 
(39) En hiver, les eaux sont assez hautes, claires et transparentes... En été, c'est nettement plus trouble. Je me souviens avoir eu vu flotter des ordures ménagères balancées depuis le restaurant « Fonze » à St Guilhem le Désert... (Blog, 2013)

\subsection{Analyses et commentaires}

\subsubsection{Type résultatif}

Ces exemples montrent que le type résultatif, qu'il concerne ou non des formes tensées, est massivement utilisé dans des contextes où il exprime la concomitance entre :

- d'une part, l'état résultant d'une situation S1 exprimé par la forme surcomposée (souvent dans une subordonnée),

- d'autre part, une situation S2 exprimée soit au moyen d'un temps simple, soit d'un temps composé à interprétation processive.

La relation établie entre S1 et S2 n'est pas qu'une relation de concomitance. L'état résultant de $\mathrm{S} 1$ fonctionne en général aussi comme un localisateur temporel de $\mathrm{S} 2$, d'où des enchaînements comme (40) :

(40) - ces accidents s'étaient manifestés peu de temps après qu'il avait eu mangé... (16) - quand celui-ci aurait eu fini de répondre, il lui aurait annoncé... (20)

- le débat a pris fin après que j'aie eu pris la parole... (29) ${ }^{11}$

- ayant eu vu le second, je me faisais une joie de regarder le premier... (33)

- après avoir eu terminé la Wirtschaftsmittelschule, il a suivi une formation... (36)

Cette fonction de localisation temporelle peut être doublée d'une relation causale. Quelques cas toutefois s'écartent un peu de cette logique temporelle. Ainsi, dans l'exemple (28) la situation désignée par la forme surcomposée indique la borne terminale d'une situation itérative (jusqu'à ce que elle ait eu fini de...). Et dans l'exemple (17), c'est en réalité l'état résultant qui fait l'objet d'une localisation temporelle, localisation indirecte puisqu'elle est signifiée par l'adverbe vite. Dans ce cas, la forme surcomposée fait progresser temporellement la narration, contrairement à ce qu'on observe dans les exemples listés en (40).

Appelons S1 situation localisante et S2 situation localisée. Le principe qui régit ces emplois est bien connu et peut être formulé comme suit ${ }^{12}$ : la forme verbale choisie pour désigner la situation localisante (typiquement, dans la subordonnée) a, sur le plan morphologique, un degré de composition de plus que celle choisie pour exprimer la situation localisée. C'est ce qu'on observe dans les exemples listés en (40). D'où les régularités résumées dans le Tableau 2.

\footnotetext{
${ }^{11}$ L'exemple (29) présente un phénomène assez curieux : la coordination, dans la subordonnée en après que, de deux propositions, la première au passé surcomposé subjonctif, la seconde au plus-que-parfait : après que j'aie eu pris la parole, et que bon nombre d'honorables sénateurs avaient posé des questions.

${ }^{12}$ Voir par exemple Damourette \& Pichon (1911-1936, tome 5, §1702).
} 
Tableau 2 : Facteurs de composition des formes verbales utilisées

pour désigner la situation localisante et la situation localisée

\begin{tabular}{lll}
\hline & $\begin{array}{c}\text { SITUATION LOCALISANTE (S1) } \\
\text { (Interprétation résultative) }\end{array}$ & $\begin{array}{l}\text { SITUATION LOCALISÉE (S2) } \\
\text { (Interprétation processive) }\end{array}$ \\
\hline \hline a fait & avoir fait, ayant fait & fait \\
\hline a eu fait & avoir eu fait, ayant eu fait & a fait (processif), faisait \\
\hline avait fait, eut fait & avoir fait, ayant fait & fit \\
\hline avait eu fait, eut eu fait & avoir eu fait, ayant eu fait & avait fait (processif) \\
\hline aura fait & avoir fait, ayant fait & fera \\
\hline aura eu fait & avoir eu fait, ayant eu fait & aura fait \\
\hline aurait fait & avoir fait, ayant fait & ferait \\
\hline aurait eu fait & avoir eu fait, ayant eu fait & aurait fait (processif) \\
\hline
\end{tabular}

Cependant, un examen plus attentif des exemples de surcomposition donnés plus haut indique que deux cas au moins doivent être distingués.

- Dans le premier, la surcomposition s'explique par une stricte application du principe ci-dessus. Si une S2 exprimée par un temps non composé est localisée par une S1 exprimée par un temps composé, alors une S2 exprimée par un temps composé doit être localisée par une S1 exprimée par un temps surcomposé. L'utilisation d'une forme surcomposée est donc ici motivée par un principe qui relève de la logique aspectuo-temporelle.

A cet égard, des enchaînements comme (41) apparaissent comme des anomalies aspectuo-temporelles, bien qu'on les rencontre assez souvent, en particulier dans l'oral ordinaire (Carruthers 1993) :

(41) Et puis, le soir, quand on en a fini avec les Allemands, je suis descendu vers la Volga. (A. Makine, 1995)

Ce type d'enchaînement oblige en effet à interpréter le passé composé de la subordonnée comme signifiant un état résultant dans le passé - ce que ce temps verbal est en principe inapte à exprimer! La forme normale attendue ici (i.e. respectant les propriétés aspectuo-temporelles des formes composées) est quand on en a eu fini, comme l'ont noté de nombreux grammairiens et linguistes. ${ }^{13}$

- Dans le second cas, la forme surcomposée (décrivant la situation localisante) est mise en correspondance avec une forme non composée (décrivant la situation localisée). Les exemples (24), (25) et (33) illustrent ce cas de figure: dans (24), aura eu fait est mis en connexion temporelle avec un impératif présent; dans (25), aura eu défilé est mis en connexion avec un futur simple; et dans (33), ayant eu $v u$ est mis en connexion avec un imparfait. Il est difficile ici d'évoquer la logique aspectuo-temporelle pour expliquer la présence d'une forme surcomposée. Les futurs surcomposés aura eu fait et aura eu défilé de (24) et (25) pourraient être remplacés, apparemment sans modification sémantique, par des futurs antérieurs; de même, ayant eu vu dans (33) pourrait être remplacé par ayant vu. En conséquence, je formulerai l'hypothèse que dans ces exemples, la surcomposition a pour seule fonction de renforcer l'interprétation résultative de la forme verbale.

Les formes du type aura eu fait (futur surcomposé) posent par ailleurs un problème spécifique, qui est le suivant. Selon le principe évoqué plus haut, ces formes devraient être utilisées pour désigner une situation S1 qui localise une situation S2 exprimée au moyen d'un

\footnotetext{
${ }^{13}$ Ce type de conflit aspectuo-temporel est fréquent dans L'Étranger de Camus où, comme on sait, l'auteur a délibérément choisi de ne pas utiliser le passé simple, mais le passé composé, pour conduire le récit principal. L'exemple (41) est d'autant plus surprenant que le verbe finir est, avec quelques autres verbes phasiques, l'un des plus fréquemment employés au passé surcomposé. Sur ces conflits aspectuo-temporels engendrés par le passé composé dans les subordonnées temporelles, voir Apothéloz (à par.).
} 
futur antérieur, ce futur antérieur ayant une interprétation processive. Or, ce temps verbal en emploi purement processif (aoristique), sans signification additionnelle - comme celle qu'on rencontre dans les emplois conjecturaux ou rétrospectifs (dits aussi de bilan) -, est réputé extrêmement rare ${ }^{14}$. Ainsi, mes corpus ne comportent aucun exemple dans lequel on aurait une subordonnée temporelle avec une forme du type aura eu fait, enchaînant sur une principale avec une forme du type aura fait interprétée processivement. Les exemples trouvés attestent quatre types d'enchaînements :

(i) soit une forme de type aura eu fait enchaînant avec un futur simple, comme dans l'exemple suivant (voir aussi l'exemple (25) ci-dessus) :

(42) Une fois qu'on aura eu fini cette tournée Quadrophenia, à Paris et à Amsterdam, les fans allemands, danois, italiens, japonais et australiens nous réclameront. (Interview du guitariste Pete Townshend, site du Figaro, 2013)

Dans ce cas la forme surcomposée ne paraît pas avoir d'autre raison d'être que de renforcer l'interprétation résultative.

(ii) soit une forme de type aura eu fait enchaînant avec un futur antérieur, ce dernier étant interprété résultativement (accompli), comme dans (43) :

(43) La manne, comme toutes les autres substances douces, aura perdu son goût doux d'abord qu'on en aura eu séparé l'acide \& l'huile qu'elle contenoit [...]. (Lamery, Cours de chymie, 1730. Corpus M. Borel $)^{15}$

Dans cet extrait, ce sont ainsi deux états résultants qui sont mis en concomitance temporelle : 'ne plus avoir son goût doux' et 'l'acide et l'huile être séparés'. Mais, étant donné que le second état est la cause du premier état, il subsiste une idée d'antériorité du second sur le premier. C'est précisément cette idée d'antériorité qu'exprime ici la forme surcomposée, qui est ainsi temporellement et aspectuellement motivée.

(iii) soit une forme de type aura eu fait enchaînant avec un futur antérieur processif, mais dans un contexte où l'énoncé tout entier a une valeur conjecturale, comme dans (44) :

(44) La rentrée de la Chambre en pleine conférence de La Haye oblige le personnel politique à de singuliers tours de force qui sont presque des miracles d'ubiquité. On pense que M. Tardieu en aura eu fini hier soir avec les résistances du Dr Schacht, il aura pris le train de 20 heures pour être à $6 \mathrm{~h} .30$ à Paris, tenir à 10 le conseil des ministres, faire l'ouverture de la Chambre à 2 heures et repartir à la minuit suivante pour le pays des sanctions et des abandons. (Ch. Maurras, 1930, in : Damourette \& Pichon 1911-1936, t.5, §1859)

Dans cet exemple, où la forme surcomposée n'est pas dans une subordonnée temporelle, l'énonciateur attribue à un 'on' impersonnel des hypothèses concernant des faits passés (cf. on pense que... hier soir) et donc révolus. Le trait 'futur' fonctionne ici comme un marqueur de conjecture.

(iv) soit une forme de type aura eu fait utilisée dans un contexte omnitemporel, c'est-àdire où la distinction des époques n'est pas pertinente: contexte générique, texte de règlement, séquence de consignes, par exemple :

(45) Après que le mari aura eu employé la douceur, puis la coercition, si l'épouse persiste dans sa désobéissance ou que l'incompatibilité d'humeur soit bien établie, on ne doit pas hésiter. (J. Sicard, Le monde musulman dans les possessions françaises. 1928, p. 45)

\footnotetext{
${ }^{14}$ Certains linguistes (e.g. Sten 1952 ; Vet 2010) considèrent même que les emplois purement processifs du futur antérieur n'existent pas.

${ }^{15}$ En français classique, d'abord que signifie 'aussitôt que', 'dès que'.
} 
Dans ce cas, à nouveau la forme surcomposée ne paraît pas avoir d'autre raison d'être que de renforcer l'interprétation résultative. L'exemple (24) ci-dessus, extrait d'une séquence de consignes, relève de cette même catégorie.

\subsubsection{Type existentiel}

Concernant le type existentiel (interprétation de parfait d'expérience), le problème qui sera examiné ici est celui de la concurrence entre les formes surcomposées et les formes composées simples. La principale question qu'on posera est la suivante : est-ce que, dans les exemples de type existentiel ci-dessus, la forme composée simple correspondante serait ambiguë, c'est-à-dire pourrait être interprétée aussi bien comme un parfait d'expérience que comme désignant une situation singulière à localisation définie ? Si c'est le cas, alors on considérera que cela suffit à justifier le choix d'une forme surcomposée.

Cet exercice de substitution conduit aux observations suivantes :

- Une forme composée simple serait de toute manière nécessairement interprétée comme un parfait d'expérience chaque fois que le verbe est sous l'incidence d'un adverbial quantifieur de fréquence ou d'occurrence. Ce cas se présente dans de nombreux exemples : très rarement dans (19), jamais dans (22), le nombre de fois où dans (26), souvent dans (27). À quoi il faut ajouter déjà à valeur existentielle dans (34).

- Une forme composée simple serait également interprétée comme un parfait d'expérience quand l'énoncé comporte une expression pouvant être interprétée comme désignant l'intervalle de validation. Il en va ainsi dans l'exemple (38), avec l'expression ces dernières années : la prédication avoir fait des injections de Botox ces dernières années n'a pas d'autre interprétation qu'existentielle. Le même constat s'applique à (26) et (27), avec les expressions dans ma vie et tout au long de leurs vies, tout à fait caractéristiques de la phraséologie des parfaits d'expérience.

- Il en va de même quand la forme composée simple concerne le tour impersonnel il est arrivé (ou un tour équivalent), qui est une forme explicite, analytique du parfait d'expérience. C'est ce qu'on observe dans l'exemple (19), où le tandem adverbial très rarement suffit d'ailleurs à sélectionner cette interprétation.

- Parfois, la signification même de l'expression verbale, ou le contexte sémantique dans lequel elle se trouve, paraissent exclure toute interprétation autre que celle de parfait d'expérience. On voit mal, par exemple, comment la formulation que des anciens l'aient fait dans l'exemple (30), où il est question de différentes pratiques concernant la façon de recharger une arme à feu, pourrait être interprétée autrement que comme un parfait d'expérience, notamment à cause de l'indéfini pluriel (des anciens). On notera que cet extrait comporte un second parfait d'expérience réalisé, lui, au moyen d'un passé composé existentiel (Moi j'ai aussi connu des poudreux qui rechargeaient...). C'est donc ici toute la séquence retenue qui est sur le mode du parfait d'expérience. Cette observation conduit inévitablement à s'interroger sur les conditions d'emploi respectives des formes composées et surcomposées pour exprimer le parfait d'expérience.

- Une forme composée simple serait également interprétée comme un parfait d'expérience dans les exemples (34) et (35). Cela est dû au fait que le complément du verbe y est une expression indéfinie. Ainsi, même sans l'adverbe déjà, le participe ayant fait dans (34) serait nécessairement interprété comme un parfait d'expérience du seul fait de l'indéfini pluriel du complément plusieurs essais négatifs. Il en va de même pour (35) (ayant fait des traumatismes similaires). Ce lien entre l'indéfini (et plus particulièrement l'indéfini pluriel) et l'interprétation de parfait d'expérience a été mis en évidence par Karolak (2007) et Mittwoch (2008).

Il n'est bien sûr pas possible d'attribuer une quelconque représentativité statistique à ces analyses par substitution. Elles indiquent au mieux que dans un nombre relativement 
important de cas, la forme surcomposée, comparée à la forme composée simple, ne présente aucune réelle nécessité sémantique, et paraît davantage s'expliquer par le besoin de marquer formellement, c'est-à-dire par une forme spécifique et dédiée, la valeur de parfait d'expérience. Cela confirme que, pour les sujets parlants dont la grammaire inclut la surcomposition comme marqueur du type existentiel, les formes surcomposées grammaticalisent cette valeur.

Mais les formes de type aura eu fait présentent, ici encore, une particularité : on pourrait, à signification pratiquement équivalente, leur substituer non seulement un futur antérieur, mais aussi un passé composé ! Le choix d'une forme du futur, dans ces exemples, produit un effet sémantique qui, s'agissant du futur antérieur, est connu sous le nom de futur antérieur de bilan, ou rétrospectif. Ce même effet se retrouve avec la forme surcomposée, et il existe donc également un futur surcomposé de bilan. L'analyse qu'on donne généralement de ces cas consiste à dire qu'il y a alors projection dans le futur du moment où sera acquise la vérité de ce qui est asserté. Autrement dit, le composant 'futur' du temps verbal ne localise plus la situation proprement dite, mais la validation de celle-ci ${ }^{16}$. Cet effet, cela va de soi, disparaît complètement avec le passé composé.

Pour terminer sur ce point, il est intéressant de signaler que certaines formulations, comme pour avoir eu + PARTICIPE PASSÉ, déclenchent quasi mécaniquement une lecture de parfait d'expérience, comme l'a noté Borel (à par.). En voici un exemple :

(46) [Il est question d'un type de phare de voiture]

Pour en avoir eu monté sur celle [ $=$ la voiture $]$ d'un ami, je te déconseille de mettre ces niaiseries. (Forum internet, 2018)

\section{Conclusion}

On sait depuis longtemps que les formes surcomposées du français manifestent une certaine diversité d'emplois, et que cette diversité présente une dimension géographique. D'assez nombreuses publications montrent que, quelles que soient les analyses et les typologies proposées, certains de ces emplois se révèlent difficiles à décrire sur le plan sémantique, tout particulièrement les emplois existentiels, qui ont longtemps été présentés comme régionaux et exprimant un passé lointain et révolu, voire une certaine nostalgie.

Le présent article est parti d'une analyse consistant à distinguer deux types majeurs de passés surcomposés: un type résultatif et un type existentiel (ce dernier réalisant une interprétation connue sous l'appellation de parfait d'expérience). Le but était, dès lors, d'évaluer le degré de généralité de ces deux types dans le système de la surcomposition verbale du français.

Les données collectées montrent que ces deux interprétations de la surcomposition présentent un degré très élevé de généralité et de systématicité. Elles permettent de décrire l'immense majorité des emplois des formes surcomposées répertoriées en français.

Dans le cas du type résultatif, les données montrent que la présence d'une forme surcomposée peut obéir à deux motivations, selon l'environnement temporel dans lequel la forme est utilisée. Quand elle est couplée avec un temps composé simple, la forme surcomposée s'explique par une stricte logique aspectuo-temporelle, de sorte que sa motivation est en quelque sorte systémique. En revanche, quand elle est couplée avec un temps non composé, il n'est plus possible d'expliquer sa présence en évoquant la logique aspectuo-temporelle; la seule explication est qu'elle permet alors de renforcer l'expression de la résultativité.

\footnotetext{
${ }^{16}$ Pour une interprétation énonciative de ce phénomène, voir Barceló \& Bres (2006) et Azzopardi \& Bres (2011)
} 
Dans le cas du type existentiel, les formes surcomposées ont, pour les sujets parlant qui les possèdent, un statut d'overt category au sens de Whorf (1956), c'est-à-dire de formes spécifiquement dédiées à l'expression de cette valeur, et qui la grammaticalisent. Elles permettent notamment d'éviter d'éventuelles ambiguïtés du temps composé simple, dont les emplois de parfait d'expérience en français ne sont qu'une covert category.

Cependant, les formes surcomposées du français présentent une différence importante avec les formes composées simples. Alors que ces dernières oscillent entre trois interprétations (résultative, processive, existentielle), les formes surcomposées oscillent entre deux interprétations (résultative, existentielle). Elles semblent donc avoir échappé, du moins pour l'instant, au phénomène de dérive aoristique qui a frappé les temps composés simples du français, qui ont depuis longtemps déjà stabilisé des emplois processifs (aoristiques). Cela dit, les emplois existentiels des temps surcomposés marquent peut-être une première phase d'un éventuel processus d'aoristisation, processus décrit par beaucoup de linguistes comme une tendance générale voire universelle affectant les parfaits (cf. Kuryłowicz 1977 ; Dahl 1985 ; Bybee et al. 1994).

\section{Références}

Apothéloz, Denis. 2010. Le passé surcomposé et la valeur de parfait existentiel. Journal of French Language Studies 20(2). 105-126.

Apothéloz, Denis. 2012. La concurrence du passé composé et du passé surcomposé dans l'expression de la valeur de parfait d'expérience. In : de Saussure, Louis \& Rihs, Alain (éds), Études de sémantique et pragmatique françaises, 39-65. Berne : Peter Lang.

Apothéloz, Denis. 2017. Le parfait d'expérience et l'évolution de la relation passé composé passé simple. In: Prévost, Sophie \& Fagard, Benjamin (éds), Le français en diachronie: Dépendances syntaxiques, morphosyntaxe verbale, grammaticalisation, 157-188. Berne : Peter Lang.

Apothéloz, Denis. À par. À propos d'un conflit aspectuo-temporel engendré par certains usages du passé composé. In : Corre, Eric, Do-Hurinville, Danh-Thành \& Dao, HuyLinh (éds), Autour de L'Étranger de Camus et de ses traductions: Approches linguistiques des questions de temps, d'aspect, de modalité et d'évidentialité. Amsterdam : J. Benjamins.

Azzopardi, Sophie \& Bres, Jacques. 2011. Temps verbal et énonciation. Le conditionnel et le futur en français : l'un est dialogique, l'autre pas (souvent). Cahiers de praxématique 56. 53-76.

Barceló, Gérard J. \& Bres, Jacques. 2006. Les temps de l'indicatif en français. Paris : Ophrys.

Beauzée, Nicolas. 1765. Article Tems. In: Encyclopédie ou Dictionnaire raisonné des sciences, des arts et des métiers, Tome 16, 96-117. Neufchastel : Chez Samuel Faulche.

Borel, Marine. 2017. Apparition et évolution du passé surcomposé en français. In : Prévost, Sophie \& Fagard, Benjamin (éds), Le français en diachronie: Dépendances syntaxiques, morphosyntaxe verbale, grammaticalisation, 189-215. Berne : Peter Lang.

Borel, Marine. À par. Les formes verbales surcomposées en français. Fribourg : Université de Fribourg \& Nancy : Université de Lorraine. (Thèse de doctorat.)

Bybee, Joan, Perkins, Revere \& Pagliuca, William. 1994. The evolution of grammar. Tense, aspect and modality in the languages of the world. Chicago: The Chicago University Press.

Caron, Philippe \& Liu, Yu-Chang. 1999. Nouvelles données sur la concurrence du passé simple et du passé composé dans la littérature épistolaire. L'Information grammaticale 82. 38-50. 
Carruthers, Janice. 1993. Passé composé, passé surcomposé : marqueurs de l'antériorité en français parlé. In: Hilty, Gerold (coord.), Actes du XX congrès international de linguistique et de philologie romane, vol. 1, 109-122. Tübingen : Francke.

Carruthers, Janice. 1994. The passé composé régional: towards a definition of its function in contemporary spoken French. Journal of French Language Studies 4(2). 171-190.

Carruthers, Janice. 1998. Surcomposé 'général' et surcomposé 'régional': deux formes distinctes? In: Ruffino, Giovanni (ed.), Atti del XXI congresso internazionale di linguistica e filologia romanza, vol. 2, 143-154. Tübingen: Max Niemeyer.

Carruthers, Janice. 1999. A problem in sociolinguistic methodology: investigating a rare syntactic form. Journal of French Language Studies 9(1). 1-24.

Caudal, Patrick \& Vetters, Carl. 2007. Passé composé et passé simple : sémantique diachronique et formelle. In : Labeau, Emmanuelle, Vetters, Carl \& Caudal, Patrick (éds), Sémantique et diachronie du système verbal français, 121-151. Amsterdam: Rodopi (Cahiers Chronos 16).

Comrie, Bernard. 1976. Aspect. Cambridge: Cambridge University Press.

Cornu, Maurice. 1953. Les formes surcomposées en français. Berne : Francke.

Croft, William. 2012. Verb: Aspect and causal structure. Oxford: Oxford University Press.

Dahl, Östen. 1985. Tense and aspect systems. Oxford: Blackwell.

Damourette, Jacques, Pichon, Edouard. 1911-1936. Des mots à la pensée. Essai de grammaire de la langue française. Paris : d'Artrey.

De Saussure, Louis \& Sthioul, Bertrand. 2012. Formes et interprétations du passé surcomposé : unité sémantique d'une variation diatopique. Langages 188. 75-94.

Desclés, Jean-Pierre. 1997. Logique combinatoire, topologie et analyse aspecto-temporelle. Études cognitives / Studia Kognitywne 2. 37-69.

Desclés, Jean-Pierre. 2017. Invariants des temps grammaticaux et référentiels temporels. Verbum 39(1). 155-189.

Fenn, Peter. 1987. A semantic and pragmatic examination of the English perfect. Tübingen: Gunter Narr.

Foulet, Lucien. 1925. Le développement des formes surcomposées. Romania 51. 203-252.

Gosselin, Laurent. 2017. Les temps verbaux du français : du système au modèle. Verbum 39(1). 31-69.

Haß, Norman. 2016. Doppelte Zeitformen im Deutschen und Französischen. Hamburg: Buske Verlag.

Havu, Jukka. 2013. Le passé surcomposé en français classique et moderne. In : SulletNylander, Françoise, Engel, Hugues \& Engwall, Gunnel (éds), La linguistique dans tous les sens, 37-58. Stockholm: Kungl. Vitterhetsakademiens Publications, Konferenser 80.

Holtus, Günter. 1995. Zur Verbreitung der formes surcomposées in den romanischen Sprachen. In: Dahmen, Wolfgang, Holtus, Günter, Kramer, Johannes, Metzeltin, Michael, Schweickard, Wolfgang \& Winkelmann, Otto (éds), Konvergenz und Divergenz in den romanischen Sprachen, 85-114. Tübingen : Gunter Narr.

Imbs, Paul. 1960. L'emploi des temps verbaux en français moderne. Paris : Klincksieck.

Jolivet, Rémy. 1986. Le passé surcomposé : emploi «général» et emploi « régional». Examen des insertions dans le syntagme verbal surcomposé. In: Mélanges d'onomastique, linguistique et philologie offerts à M. Raymond Sindou, vol. 2, 109116. Bâle : Centre du FEW.

Karolak, Stanisław. 2007. Sktadnia francuska o podstawach semantycznych [Syntaxe française à bases sémantiques], Tom 1. Kraków: Collegium Columbinum.

Kuryłowicz, Jerzy. 1977. Problèmes de linguistique indo-européenne. Wrocław, Warszawa, Kraków : Ossolineum.

Leech, Geoffrey N. 1971. Meaning and the English verb. London: Longman. 
McCawley, James D. 1971. Tense and time reference in English. In: Fillmore, Charles J. \& Langendoen, D. Terence (éds), Studies in Linguistic Semantics, 96-113. New York: Holt, Rinehart and Winston.

McCawley, James D. 1981. Notes on the English perfect. Australian Journal of Linguistics 1. 81-90.

Meigret, Louis. 1550. Le tretté de la grammere françoeze. Paris : Chrestien Wechel. Réédité par Wendelin Foerster, Heilbronn Verlag, 1888.

Melchior, Luca. 2012. Tra esperienzialità e iteratività : il 'passé surcomposé à valeur spéciale' in francese (e in altri idiomi romanzi). Revue de linguistique romane 76. 65-98.

Michaelis, Laura A. 1998. Aspectual grammar and past-time reference. London: Routledge.

Mittwoch, Anita. 2008. The English Resultative perfect and its relationship to the Experiential perfect and the simple past tense. Linguistics and Philosophy 31. 323-351.

Olsson, Lars. 1971. Etude sur l'emploi des temps dans les propositions introduites par quand et lorsque et dans les propositions qui les complètent en français contemporain. Uppsala : Tofters-Wretmans boktryckeri.

Paesani, Katherine A. 2001. The syntax and semantics of the passé surcomposé in modern French. Bloomington : Indiana University. (Doctoral dissertation.)

Schaden, Gerhard. 2009. Composés et surcomposés. Paris : L'Harmattan.

Schøsler, Lene. 2012. Sur l'emploi du passé composé et du passé simple. In : Guillot, Céline, Combettes, Bernard, Lavrentiev, Alexei, Oppermann-Marsaux, Evelyne \& Prévost, Sophie (éds), Le changement en français. Études de linguistique diachronique, 321339. Berne : Peter Lang.

Squartini, Mario. 1998. Verbal periphrases in Romance: aspect, actionality, and grammaticalization. Berlin, New York: Mouton de Gruyter.

Stefanini, Jean. 1954. La tradition grammaticale française et les temps surcomposés. Annales de la Faculté des lettres d'Aix 28. 67-108. Réédité dans: Linguistique et langue française. Textes de J. Stefanini réunis par P. Bonnefois, 37-74. Paris : Editions du CNRS, 1992.

Sthioul, Bertrand. 2006. Le(s) passé(s) surcomposé(s) : temps, aspect, subjectivité. Travaux neuchâtelois de linguistique (TRANEL) 45. 115-132.

Sten, Holger. 1952. Les temps du verbe fini (indicatif) en français moderne. Kobenhavn: Munksgaard.

Vet, Co. 2010. L'interprétation des formes composées. In : Flaux, Nelly, Stosic, Dejan \& Vet, Co (éds), Interpréter les temps verbaux, 11-31. Berne : Peter Lang.

Vetters, Carl. 2010. Développement et évolution des temps du passé en français : passé simple, passé composé et venir de + infinitif. In : Moline, Estelle \& Vetters, Carl (éds), Temps, aspect et modalité en français, 277-298. Amsterdam: Rodopi (Cahiers Chronos 21).

Wagner, Robert-Léon. 1954. Compte rendu de Les formes surcomposées en français de M. Cornu. Bulletin de la société de linguistique de Paris 50. 97-100.

Whorf, Benjamin Lee. 1956. Language, thought and reality. Cambridge (Mass.): The M.I.T. Press. Traduction française : Linguistique et anthropologie. Paris : Denoël/Gonthier, 1969.

Wilmet, Marc. 2009. Le passé surcomposé sous la loupe. Journal of French Language Studies 19(3). 381-399.

Zandvoort, Reinard W. 1932. On the Perfect of Experience. English Studies 14. 11-20, 7679. 